\title{
CONCRETE MIXING FOR GORILLAS
}

Corning's Gorilla Glass must be one of the great success stories in modern materials. Pretty much everyone with a mobile phone, tablet or other touchscreen device benefits from this amazingly robust, scratch-proof glass (or one of its competitors). Provided you don't drop your phone onto a stone floor, it feels close to being an immortal material.

Chemically strengthened glass dates back to the 1960s, when it was in demand for the automotive and aerospace industries. But it was touchscreen electronics that made the difference. Famously, Corning is said to have been pushed into its breakthrough by Apple's Steve Jobs, who, searching for the right material for the iPhone concept in 2006, told the company "Get your mind around it. You can do it." With Apple on board, Gorilla Glass couldn't fail (almost literally).

In 2013 Corning introduced Gorilla Glass 3, which was still more scratch-resistant. Allegedly, the next-generation Gorilla Glass 4 will even withstand being dropped onto a hard surface. But perhaps the most remarkable thing about Gorilla Glass 3 is that it was designed at the drawing board, so to speak, rather than the lab. It is hardened by getting just the right chemical composition of elements in the alkali aluminosilicate, with some of the sodium ions being replaced with potassium. To predict that ideal composition, Corning scientists used rigidity theory, an approach first developed by the pioneer of glass microstructural physics James Phillips in the late $1970 \mathrm{~s}^{1}$.

In rigidity theory, the mechanical properties of a glass are calculated from the topology of the atomic network, which is disorderly at long range but has some structural order at short range. The network is regarded rather like a mechanical truss, and its flexibility or rigidity is deduced from the constraints on the movements of atoms - by stretching or bending of bonds - due to their links to neighbouring atoms. Loosely speaking, these constraints can give rise to networks that are floppy, locked rigid, or somewhere in between. By changing the chemical composition of the network, a glass can undergo a rigidity transition that renders it extremely hard and scratch-resistant.

This is all very useful, as Gorilla Glass testifies - which is why it would be good to know if rigidity theory (also called topological constraint theory) can be applied to a wider range of materials than glasses. Bauchy et al. report evidence that it $\mathrm{can}^{2,3}$. They have used the same concepts of topological constraints to look at the rigidity of the calcium

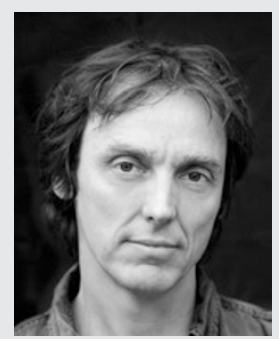

PHILIP BALL

silicate hydrate network that forms the binding phase of concrete. Here too they see a rigidity transition as a function of the $\mathrm{Ca} / \mathrm{Si}$ ratio of the composition: the first direct evidence of such a thing in a non-glassy material. The bond-stretching and bondbending constraints play different roles in contributing to the hardness: this property depends mostly, and linearly, on the number of bending constraints (which are weak compared with stretching) per atom. While concrete is hardest for low $\mathrm{Ca} / \mathrm{Si}$ ratios, it is - like glasses - toughest for the intermediate state between rigid and flexible. With such information to hand, maybe concrete too can be designed from theory alone.

References

1. Phillips, J. C. J. Non-Cryst. Solids 34, 153-181 (1979).

2. Bauchy, M. et al. J. Phys. Chem. C

118, 12485-12493 (2014).

3. Bauchy, M. et al. Phys. Rev. Lett. 114, 125502 (2015). 